\title{
Medicine detection with Low Frequency Electromagnetic Signals
} ERIETTA VASILAKI AND EMMANOUEL ANTONIDAKIS

\author{
Department of Electronic Engineering \\ Hellenic Mediterranean University \\ Romanou 3, Chania 73133 \\ GREECE
}

\begin{abstract}
This paper presents a new, non-invasive method to detect molecular structures inside materials. There have been different methods to detect molecular structures such as Chromatography, Spectroscopy and Nuclear Magnet Resonance. A brief description of these methods is presented and how they are used. Then the new method is presented using an apparatus emitting low frequency electromagnetic signals. This paper presents how the frequencies are found, that are used to detect molecular structures. The method was applied for finding the frequencies of four pain-reliever medication. Discussion follows on the results. Conclusions are drawn and further work is proposed.
\end{abstract}

Keywords: - Detection with Low Frequency Signals, Pain-Relief Medication Detection, Molecular Structure Detection, Non-Invasive Detection

Received: January 23, 2020. Revised: August 28, 2020. Re-revised: September 15, 2020. Accepted: September 30, 2020. Published: October 20, 2020.

\section{Introduction}

The aim of this research is on the detection of chemical substances inside materials using low frequency electromagnetic signals. More specifically the research involves the scanning of chemical substances and the development of a noninvasive method, that will find the frequencies that each material responds. Application on the detection of materials will be depicted and examples will be presented. This method has already successfully been used for the detection of molecular structures, such as pain-relievers medication. It could also be applied for the detection of other chemical structures that reside inside materials.

Until now, methods such as Chromatography, Ultraviolet-Visible spectroscopy, Nuclear Magnetic Resonance (NMR), were used to analyze presence of chemical structures inside different substances.

\section{Background}

To identify various chemical structures inside a material, there are different techniques used, such as Chromatography, Ultraviolet-Visible Spectroscopy and Nuclear Magnetic Resonance. They are briefly described below:

\subsection{Chromatography}

Chromatography is a technique to detect the constituents of a mixture. The mixture is dissolved in a fluid called the mobile phase, which carries it through a structure holding another material called the stationary phase. The various constituents of the mixture travel at different speeds, causing them to separate. The separation is based on differential partitioning between the mobile and stationary phases. Subtle differences in a compound's partition result in different retention on the stationary phase and thus affect the separation.

Chromatography may be preparative or analytical. The purpose of preparative chromatography is to separate the components of a mixture for later use, and is thus a form of purification. Analytical chromatography is done normally with smaller amounts of material and is for establishing the presence or measuring the relative proportions of analytes in a mixture. [1]

\subsection{Ultraviolet-Visible Spectroscopy (UV- Vis)}

Ultraviolet-visible spectroscopy or ultravioletvisible spectrophotometry (UV-Vis or UV/Vis) refers to absorption spectroscopy or reflectance spectroscopy in part of the ultraviolet and the visible and adjacent spectral regions. The absorption or reflectance in the visible range directly affects the perceived color of the chemicals involved. In this region of the electromagnetic spectrum, atoms and molecules undergo electronic transitions. Absorption spectroscopy is complementary to fluorescence spectroscopy, in that fluorescence deals with transitions from the excited state to the ground state, while absorption measures transitions from the ground state to the excited state.

The instrument used in ultraviolet-visible spectroscopy is called a UV/Vis spectrophotometer. It measures the intensity of light after passing through a sample and compares it to the intensity of light before it passes through the sample. [2] 


\subsection{Nuclear Magnetic Resonance (N. M. R.)}

Nuclear magnetic resonance spectroscopy is a spectroscopic technique to observe local magnetic fields around atomic nuclei. The sample is placed in a magnetic field and the NMR signal is produced by excitation of the nuclei sample with radio waves into nuclear magnetic resonance, which is detected with sensitive radio receivers. The intramolecular magnetic field around an atom in a molecule changes the resonance frequency, thus giving access to details of the electronic structure of a molecule and its individual functional groups. These fields are unique and highly characteristic to individual compounds. The NMR spectroscopy is the definitive method to identify monomolecular organic compounds. Similarly, biochemists use NMR to identify proteins and other complex molecules. Besides identification, NMR spectroscopy provides detailed information about the structure, dynamics, reaction state, and chemical environment of molecules. The most common types of NMR are proton and carbon-13 NMR spectroscopy and it is applicable to any kind of sample that contains nuclei possessing spin. [3][8]

\section{Detector System Apparatus and Method}

An apparatus is shown that uses low frequencies on a rotating antenna. The antenna is on an inclined free circular motion.

\subsection{Detector System Apparatus}

The apparatus used and its operation is briefly presented and is shown on figure 1. The apparatus consists of a flat base with an inclined pole and a rectangle box with a signal generator circuitry and an antenna. The rectangle box with the antenna can rotate on the pole. The antenna is emitting a low frequency signal, and is in an inclined circular motion. It has been observed that under some circumstances a force is exerted on the rotating antenna in the presence of a material. The force appears when a material is pointed by the antenna while the antenna is emitting a specific signal for that material. The presence of the force can be used for the detection of materials.

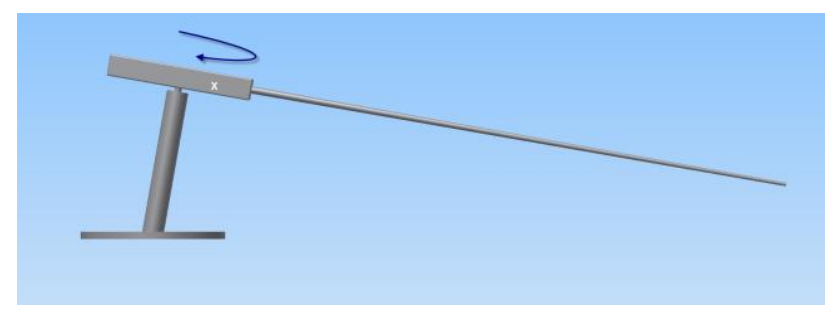

Fig. 1 Detector Apparatus
The antenna is emitting appropriate signal for the specific material. The method how the appropriate signals are found for each specific material or family of materials is presented. Some parameters that affect the force will be analysed.

The presence of the Force designates a response from the material. When the material is removed, then the Force disappears. When the material is moved to another position, then the Force appears when the antenna is pointing to the new position.

The system has a rest position when the antenna is pointing downwards. This position is 180 degrees from the highest position located exactly opposite. The highest position is defined to be at 0 degrees. The system is brought to an initial position/angle at $\varphi_{i}$ (i.e at 30 degrees) and released. The system does a specific movement. It rotates until it reaches its final position/angle $\varphi_{F}$ (i.e. at 320 degrees). The characteristics of the movement are noted, such as the position of the antenna in time. If during a rotation, a delay is experienced in the movement, this designates the presence of material and in this case the antenna's final position $\varphi_{\mathrm{f}}$ will not reach the final position of the movement $\varphi_{F}$. The position/angle $\varphi_{\mathrm{d}}$ of the antenna, when the delay is experienced designates the detection of material and its direction is $\varphi_{\mathrm{d}}$.

There are different parameters that affect the Force exerted on the antenna. The force F, exerted on the antenna of the system, opposing to the movement, is a function (f) of: the quantity $\mathrm{M}$ of the molecular structure and the distance $\mathrm{D}$ from the material.

$$
\mathrm{F}=\mathrm{f}(\mathrm{M}, \mathrm{D})
$$

The larger the quantity of the material $\mathrm{M}$, the larger is the force F. Material is counted to be only the weight of the pure active material.

The smaller the distance $\mathrm{D}$ between the material and the antenna, the larger is the force F.

The frequencies that will be used are in the low frequency range.

\subsection{Method Finding the Detection Frequencies of Materials}

To find the frequencies that respond to a material requires a specific method and it is very laborious, and has the following steps in 2 phases A, B. During this process the parameters that affect the force exerted on the antenna, $\mathrm{M}$ and $\mathrm{D}$, must be kept constant. Response to frequencies from 2300-2500 $\mathrm{Hz}$ will be tested using the method.
A. Finding the frequencies that respond
1. The material is placed on specific angle $\varphi_{\mathrm{p}}$. 
2. The signal generator on the apparatus is emitting an initial frequency $f_{i}$.

3. Then antenna is brought to the initial angle $\varphi_{\mathrm{i}}$ and is released. If the final angle of this movement $\varphi_{\mathrm{f}}$ reaches the final angle $\varphi_{\mathrm{F}}$, then there is not detection.

4. Then the frequency emitted by the generator is increased by $1 \mathrm{~Hz}$, the antenna is brought to the initial angle, released and checked if $\varphi_{\mathrm{f}}$ reached the final angle $\varphi_{\mathrm{F}}$.

5. The previous step 4 , is repeated until the antenna does not reach the final angle $\varphi_{\mathrm{f}}$. The detected frequency $f_{d}$ is recorded.

B. Centering the responding frequencies found in $\mathrm{A}$

1. The detected frequencies $\mathrm{f}_{\mathrm{d}}$ found in $\mathrm{A}$, are checked again, one at a time, for confirmation. The antenna is brought to the initial angle $\varphi_{\mathrm{i}}$ and is released. The final angle $\varphi_{\mathrm{f}}$ is recorded for each one of them.

2. From the detected frequencies $f_{d}$, from step 1 above, that are consecutive, the one that has the smaller $\varphi_{\mathrm{f}}$ is kept. The others are discarded.

\section{Frequency response of some Pain- Reliever Medications}

The apparatus and method presented in section 3 of this paper was used to find the frequencies that some mild pain-reliever medicine respond. Discussion on the detected frequency response follows.

\subsection{Testing mild pain-relievers}

Four common mild pain-reliever medications were used with the apparatus and the method was applied. These medications are Depon, Aspirin, Nurofen and Ponstan. The characteristics of these four medications as well as their molecular structures are presented below.

\subsubsection{Paracetamol (Depon)}

Paracetamol, also known as acetaminophen, is a medication used to treat pain and fever. It is typically used for mild to moderate pain relief.

Paracetamol, brand names: Depon, Panadol, etc. [4]
Chemical formula: $\mathrm{C} 8 \mathrm{H} 9 \mathrm{NO} 2$

Molar mass: $151.165 \mathrm{~g} \cdot \mathrm{mol}-1$<smiles>CC(=O)Nc1ccc(O)cc1</smiles>

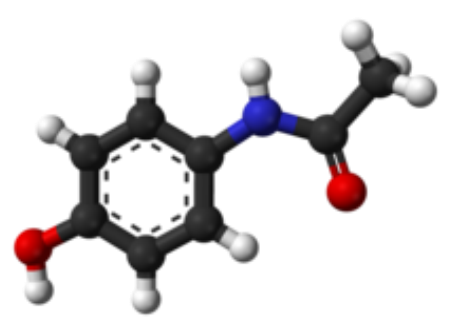

\subsubsection{Acetylsalicylic acid (Aspirin)}

Acetylsalicylic acid (ASA), is a medication used to reduce pain, fever, or inflammation. Specific inflammatory conditions which aspirin is used to treat include Kawasaki disease, pericarditis, and rheumatic fever.

Acetylsalicylic acid, brand names: Aspirin, etc.[5] Chemical Formula:C9H8O4

Molar mass: $180.159 \mathrm{~g} \cdot \mathrm{mol}-1$

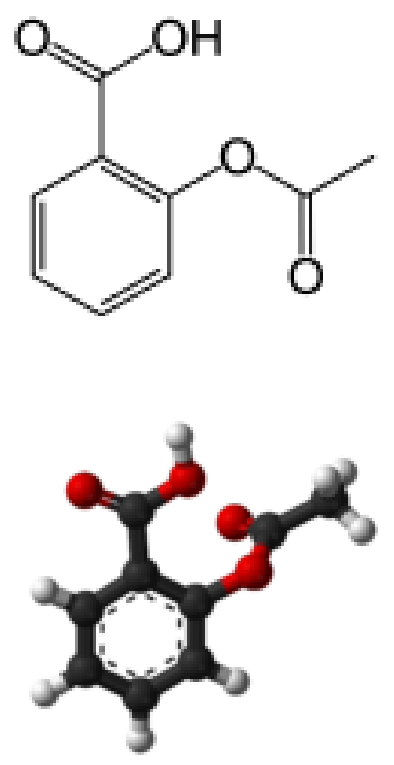

\subsubsection{Ibuprofen (Nurofen)}

Ibuprofen is a medication in the nonsteroidal antiinflammatory drug (NSAID) class that is used for treating pain, fever, and inflammation. This includes painful menstrual periods, migraines, and rheumatoid arthritis.

Ibuprofen brand names: Algofren, Seractil, Nurofen, etc. [6] 
Chemical Formula: C13H18O2

Molar mass: $206.285 \mathrm{~g} \cdot \mathrm{mol}-1$<smiles>CC(C)Cc1ccc(C(C)C(=O)O)cc1</smiles>

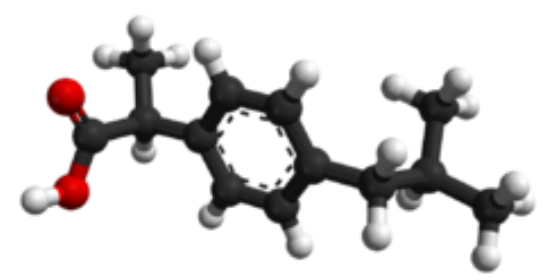

\subsubsection{Mefenamic Acid (Ponstan)}

Mefenamic acid is a member of the anthranilic acid derivatives (or fenamate) class of nonsteroidal antiinflammatory drugs (NSAIDs), and is used to treat mild to moderate pain.

Mefenamic Acid brand names: Ponstan, etc.[7]

Chemical Formula:C15H15NO2

Molar mass:241.290 g.mol-1

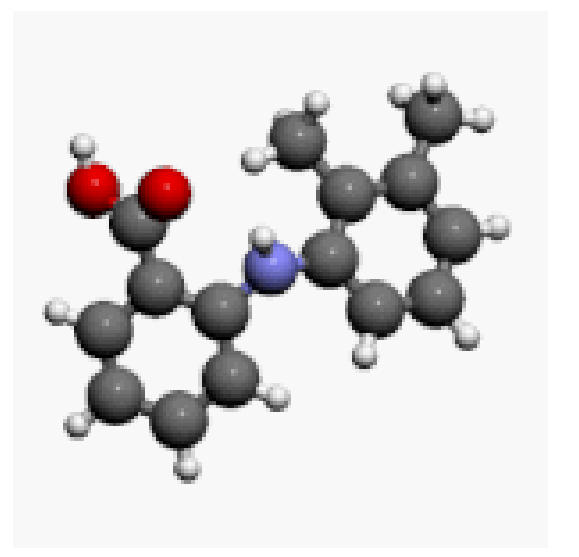<smiles>Cc1cccc(Nc2ccccc2C(=O)O)c1C</smiles>

Below is the table showing the detected frequencies. Table 1: Frequency Table: Depon, Aspirin, Nurofen and Ponstan

\begin{tabular}{|l|l|l|l|}
\hline $\begin{array}{l}\text { Depon } \\
\text { Paracetamol) } \\
2300-2500 \mathrm{~Hz}\end{array}$ & $\begin{array}{l}\text { Aspirin } \\
\text { (Acetylsalicylic } \\
\text { Acid) } \\
2300-2500 \mathrm{~Hz}\end{array}$ & $\begin{array}{l}\text { Nurofen } \\
\text { (Iburofen } \\
\text { ) }\end{array}$ & $\begin{array}{l}\text { Ponstan } \\
\text { (Mefenam } \\
2500 \mathrm{~Hz}\end{array}$ \\
$\begin{array}{l}\text { ic Acid) } \\
2300-2500 \\
\mathrm{~Hz}\end{array}$ \\
\hline 2381 & 2346 & 2395 & 2391 \\
\hline 2386 & 2429 & 2467 & 2429 \\
\hline 2391 & 2483 & 2491 & 2453 \\
\hline & 2494 & 2497 & 2486 \\
\hline
\end{tabular}

\subsection{Analysis and Discussion}

It is worth to notice that using this apparatus and the described method, we get response from materials in the range of low frequencies. Looking at the frequencies that these four medications respond, it can be observed that:

a. Aspirin and Ponstan both respond to $2429 \mathrm{~Hz}$. Also Ponstan frequency $2486 \mathrm{~Hz}$ is close to Aspirin frequency of $2483 \mathrm{~Hz}$. Ponstan is also responding at $2383 \mathrm{~Hz}$, during frequency finding phase $\mathrm{A}$, but during phase $\mathrm{B}$ was centered $2386 \mathrm{~Hz}$. Below it is seen the common part between Aspirin (left) and Ponstan (right).<smiles>COc1ccccc1C(=O)O</smiles><smiles>Nc1ccccc1C(=O)O</smiles>

The $-\mathrm{O}-$ in Aspirin is similar to Ponstan-(NH)-

b. Ponstan frequency $2391 \mathrm{~Hz}$ is close to Depon and Nurofen frequency of $2395 \mathrm{~Hz}$. Ponstan is also responding at $2395 \mathrm{~Hz}$, during frequency finding phase A, but during phase B was centered $2391 \mathrm{~Hz}$. Below it is seen the common part between Depon and Ponstan<smiles>CNc1ccc(I)cc1</smiles>

c. Aspirin frequency $2494 \mathrm{~Hz}$ is close to Nurofen frequency of $2491 \mathrm{~Hz}$ and $2497 \mathrm{~Hz}$. Aspirin is also responding at $2491 \mathrm{~Hz}$ and $2497 \mathrm{~Hz}$ during frequency finding phase $\mathrm{A}$, but during phase $\mathrm{B}$ was centered 
2494Hz. The similar part between in their molecules is shown below, Aspirin (left) and Nurofen (right)

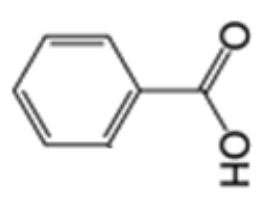<smiles>CC(C(=O)O)c1ccccc1</smiles>

d. Depon and Nurofen respond to $2390 \mathrm{~Hz}$ and $2395 \mathrm{~Hz}$ respectively, which are very close. They The similar part between in their molecules is shown below, Depon (left) and Nurofen (right)<smiles>CC(=O)Nc1ccccc1</smiles>

Studying the molecular structures of these four painrelievers, it can be noticed that they have similar parts in their molecular structure. The frequency may detect part of the molecular structure. The above a. b. c. d. observation on the similarities between the molecular structures and the detected frequency response will give insight to relate the molecular structure with frequency. The total of all the frequencies that a material is detected, is the frequency response of that material. It may be the signature that verifies the identity of the material.

\section{Conclusion}

In conclusion, an apparatus emitting electromagnetic signals was used and a method was developed to detect materials. The apparatus and method were tested on the detection of four painreliever medicine that have similarities in their molecular structures. Frequencies found that detect each of these four medications at the low frequencies range. Some frequencies are common, or very close, between these four materials. Further work needs to be done to find the relation between frequency and molecular structure.

Another aspect of work that should be looked at is if the method can be applied to find the biochemical composition of plants. With the existing methods a large sample is required which is costly in processing. Also specific ways of preserving both the samples and the substances are needed. The process has to be repeated, resulting significant delay. This new method is promising as it could detect substances inside the plants. In addition, due to the fact that it is non-invasive, the plants do not need any treatment at all, thus reducing the time of the experiment and the cost of the process. Further work needs to be done to examine this.

References:

[1] Ivor Smith, "Chromatographic and

Electro-phoretic Techniques", Volume I Chromatography

Willieam Heinemann. Medical books Lts, London 2013

[2] Marie-Christine Fournier-Salaün, Philippe Salaün, "Quantitative determination of hexavalent chromium in aqueous solutions by UV-Vis spectrophotometer", Central European Journal of Chemistry, Volume 5, pp.1084-1093, 2007.

[3] Geoffrey Bodenhausen, Ray Freeman, "Correlation of proton and carbon-13 NMR spectra by heteronuclear two-dimensional spectroscopy",Journal of Magnetic Resonance, Volume 28, Issue 3, pp. 471-476, 1977

[4] https://en.wikipedia.org/wiki/Paracetamol

[5] https://en.wikipedia.org/wiki/Aspirin

[6] https://en.wikipedia.org/wiki/Ibuprofen

[7] https://en.wikipedia.org/wiki/Mefenamic_acid

[8] Brian M. Tissue, "Nuclear Magnetic Resonance (NMR) Spectroscopy". Technische Universitaet

Braunschweig (1996)

\section{Sources of funding for research presented in a scientific article or scientific article itself}

Report potential sources of funding if there is any

\section{Creative Commons Attribution}

\section{License 4.0(Attribution 4.0 \\ International ,CC BY 4.0)}

This article is published under the terms of the Creative Commons Attribution License 4.0 https://creativecommons.org/licenses/by/4.0/deed.en US 